\section{Saul Herzt Award 2019 an Professor Richard P. Baum}

Prof. Dr. Richard P. Baum, Chefarzt der Klinik für Molekulare Radiotherapie und Präzisionsonkologie der Zentralklinik Bad Berka, ist am 24. Juni 2019 auf der Jahrestagung der SNNMI in Anaheim (Kalifornien) mit dem Saul Herzt Award 2019 ausgezeichnet worden.

Der Saul Hertz Award ehrt herausragende Leistungen in der Radionuklidtherapie und wird jährlich vergeben. Sein Namensgeber gilt als Pionier der Radiojodtherapie: Dr. Hertz behandelte 1941 erstmals eine Patientin mit Schilddrüsenüberfunktion (Morbus Basedow) mit I-131. Der 1905 geborene Hertz beeinflusste entscheidend die Ent- wicklung unseres Fachgebiets durch seine Forschung sowohl in Harvard als auch am MIT (Massachusetts Institute of Technology). In den vergangenen Jahren wurden amerikanische Wissenschaftler mit dem Preis ausgezeichnet.

Herr Professor Baum erhielt die Auszeichnung insbesondere wegen seiner Verdienste auf dem Gebiet der Theranostik (erste Radiorezeptortherapie in Deutschland 1997 sowie weltweit erste Radioligandentherapie mit Lutetium-177 PSMA im Jahre 2013) und ist der erste europäische Nuklearmediziner, der diese Auszeichnung verliehen bekommt.
VERANTWORTLICH FÜR DEN INHALT

Deutsche Gesellschaft für Nuklearmedizin e. V. (DGN)

Prof. Dr. Bernd Joachim Krause (Präsident)

Nikolaistr. 29, 37073 Göttingen

E-Mail: office@nuklearmedizin.de

Schriftführer:

Prof. Dr. Peter Bartenstein

E-Mail: office@nuklearmedizin.de 